\title{
A brief history of Regional Warning Center China (RWC-China)
}

\author{
Han He $\mathbf{H e}^{1,2,3}$, Huaning Wang ${ }^{1,2,3}$, Zhanle Du ${ }^{1,2,3}$, Xin Huang ${ }^{1,2}$, Yan Yan ${ }^{1,2}$, Xinghua Dai ${ }^{1,2}$, Juan Guo $^{1,2}$, \\ and Jialong Wang ${ }^{1,2}$ \\ ${ }^{1}$ National Astronomical Observatories, Chinese Academy of Sciences, Beijing, China \\ ${ }^{2}$ CAS Key Laboratory of Solar Activity, Chinese Academy of Sciences, Beijing, China \\ ${ }^{3}$ University of Chinese Academy of Sciences, Beijing, China \\ Correspondence: Han He (hehan@nao.cas.cn)
}

Received: 22 November 2017 - Revised: 2 March 2018 - Accepted: 8 March 2018 - Published: 27 March 2018

\begin{abstract}
Solar-terrestrial prediction services in China began in 1969 at the Beijing Astronomical Observatory (BAO), Chinese Academy of Sciences (CAS). In 1990, BAO joined the International URSIgram and World Days Service (IUWDS) and started solar-terrestrial data and prediction interchanges with other members of IUWDS. The short-term solar activity prediction service with standard URSIgram codes began in January 1991 at BAO, and forecasts have been issued routinely every weekday from then on. The Regional Warning Center Beijing (RWC-Beijing) of IUWDS was officially approved in China in 1991 and was formally established in February 1992. In 1996, the IUWDS was changed to the current name, the International Space Environment Service (ISES). In 2000, the RWC-Beijing was renamed RWC-China according to ISES requirements. In 2001, the National Astronomical Observatories, CAS (NAOC) was established. All the solar-terrestrial data and prediction services of BAO were taken up by NAOC. The headquarters of RWC-China is located on the campus of NAOC.
\end{abstract}

\section{Introduction}

Early in the 19th century, people noticed that there is a relationship between solar eruptive events and geomagnetic activity, which in turn affects electrical and electronic equipment on the Earth (Carrington, 1859; Green and Boardsen, 2006). In 1928, when solar and geophysical observations had been carried out routinely, the URSI (International Union of Radio Science) Central Committee of URSIgrams initiated rapid international information interchange services for solar-terrestrial data and solar activity predictions (URSI, 2017; ISES, 2017). The term URSIgram represents a series of predefined codes developed for quick solar-terrestrial information exchange using telex (URSIgram, 2017). The URSIgram message is still in use for its simplicity, but the telex has been replaced by e-mail.

When mankind entered the space age in the second half of the 20th century, with increasing requirements for space environment support for high technological infrastructures, solar-terrestrial monitoring and prediction services have been shown to be more important than ever. The concept of space weather was put forward (Lanzerotti, 2017). To deal with this challenge, in 1962, the International URSIgram and World Days Service (IUWDS) was formed. It is the combination of the former URSI Central Committee of URSIgrams and the former International World Days Service, which started in 1959 as a continuation of part of the International Geophysical Year (ISES, 2017). In 1996, the IUWDS was renamed to the current name, the International Space Environment Service (ISES), to reflect its function and responsibility more clearly. The mission of ISES is to organize and promote the international monitoring and prediction of space weather and help users to reduce the impact of space weather disasters (ISES, 2017).

The ISES network is formed from regional warning centers (RWCs) distributed around the world, which are institutions responsible for the interchanges of solar-terrestrial data and space weather predictions. Currently, ISES has a total of 18 RWCs from different countries (ISES, 2017). As one of the RWCs, RWC-China was established and has participated in the space weather information interchanges of ISES for 
more than 25 years. In the following sections, we give a brief description of the history of RWC-China.

\section{Early years of solar-terrestrial data and prediction services in China}

Solar-terrestrial monitoring and data services in China can be traced back to 1958, when the staff at the Beijing Astronomical Observatory (BAO) of the Chinese Academy of Sciences (CAS), together with researchers of other institutions in China, participated in the International World Days Service of the International Geophysical Year (NAOC, 2009). Solar activity prediction method and model studies began in the 1960s at BAO. In April 1969, promoted by the requirements of the first Chinese satellite mission, BAO distributed its first operational solar activity prediction in China, which included forecasts of solar proton events and shortwave fadeouts (associated with solar eruptive events) with a lead of 1 month. This so-called medium-term solar activity prediction (at first once per month, later changed to twice per month) was provided mainly for the Chinese space missions, the geomagnetic monitoring institutions and the ionospheric monitoring institutions, and has been kept as a regular service of BAO ever since. (The BAO and its solar-terrestrial prediction services were taken up by the NAOC after 2001; see Sect. 4.)

In 1971, a new journal called Chinese Solar Geophysical Data (CSGD) was founded for the solar-terrestrial prediction community in China. BAO was responsible for editing and publishing this journal. The solar-geophysical data contained in the CSGD are collected by the observatories and observing stations that belong to several institutions in China and are distributed in different locations nationwide (Wang, 1988).

In 1972, research papers on solar eruptive event predictions began to appear in Chinese domestic academic meetings. In the late 1970s, IUWDS (see the Introduction) began to organize international conferences on solar-terrestrial predictions and facilitate international cooperation in this field. Chinese researchers have attended all the international science meetings organized by IUWDS since 1979 and gradually integrated into the international community on solarterrestrial predictions. In 1980, CSGD began to participate in international solar-terrestrial data interchanges with the scientific data centers of other countries.

In the mid-1980s, there was a great improvement in the solar observation conditions at BAO. The new observing base, Huairou Solar Observing Station (HSOS), which is located at the waterside of the Huairou Reservoir in the suburb of Beijing, was completed and put into use. A new-generation solar telescope, the Solar Magnetic Field Telescope (SMFT), was set up at HSOS in 1986 and put into routine observation in 1988 (Ai, 1987; Zhang et al., 2007). Figure 1 shows the SMFT when it was just completed (NAOC, 2009). The SMFT can obtain high-quality vector magnetograms of solar

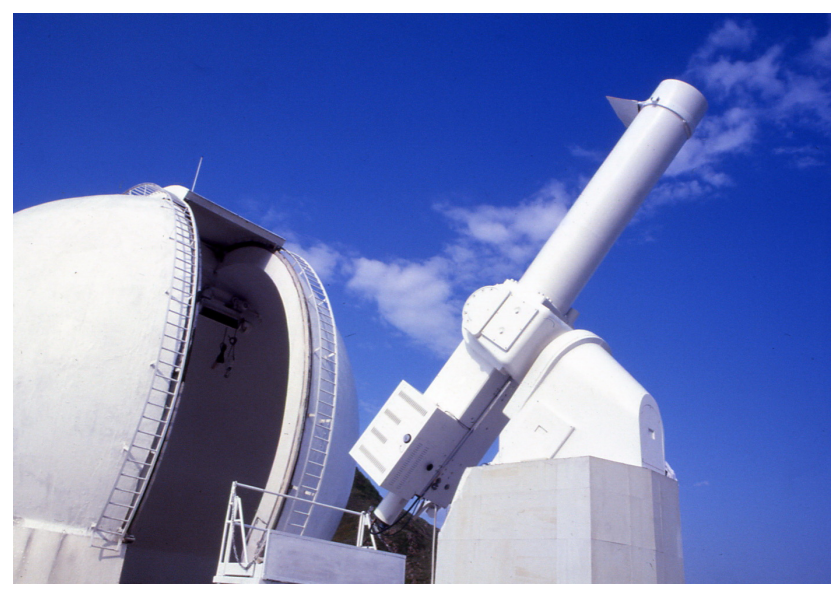

Figure 1. The Solar Magnetic Field Telescope (SMFT) at Huairou Solar Observing Station (HSOS) of BAO when it was set up in 1986. This figure is reproduced from the book by the NAOC (2009).

active regions and has maintained continuous observations until the present day. The vector magnetic field accumulated by SMFT in the past decades greatly improved solar activity prediction methods and modeling studies (He et al., 2008). The timely vector magnetograms of SMFT can also be helpful for the daily forecasting of solar eruptive events (Zhang et al., 1994). The HSOS vector magnetogram data have been included in CSGD since 1995 (Wang, 1997).

\section{Construction of RWC-Beijing}

In 1990, as the representative of the solar-terrestrial prediction community in China, BAO submitted an application to the IUWDS Steering Committee to participate in the IUWDS and set up a new regional warning center in China (NAOC, 2009). The application was accepted and agreed by IUWDS. In the same year, the Solar Activity Prediction Center (SAPC) was founded at BAO and started solarterrestrial data and prediction interchanges with other members of IUWDS. Besides BAO, the SAPC also includes the Purple Mountain Observatory (PMO) and the Yunnan Astronomical Observatory (YNAO) of CAS to form a network for solar activity monitoring and prediction in China.

In May 1991, the Regional Warning Center Beijing (RWCBeijing) of IUWDS was officially approved in China and was formally established in February 1992. RWC-Beijing consists of four subcenters; besides the SAPC at BAO, the other three subcenters are the Geophysical Prediction Center, the Space Environmental Prediction Center, and the Ionospheric Prediction Center, which belong to several different institutions in China (Wang, 1993; see Table 1). The headquarters of RWC-Beijing is located on the campus of BAO. The tasks of RWC-Beijing are improving the solar-terrestrial forecast level, enlargement of the service field, collaboration with domestic and international organizations, and exchanging fore- 
cast information and observational data with other RWCs to serve the users in local regions and the world.

Data collection and solar-terrestrial predictions at RWCBeijing were made through its four subcenters. The solarterrestrial data collected by RWC-Beijing include daily sunspot number, sunspot area, parameters of sunspot groups, $\mathrm{H} \alpha$ flares, $10.7 \mathrm{~cm}$ flux and bursts, cosmic ray intensity, geomagnetic indices and storms, and sudden ionospheric disturbances. The daily short-term solar-terrestrial predictions of RWC-Beijing include solar X-ray flare forecasts, solar proton event forecasts, and geomagnetic storm forecasts (Wang, 1997). BAO was responsible for receiving, combining, and archiving these materials and producing the standard daily URSIgram messages of RWC-Beijing. The solar-terrestrial data collected were published in the CSGD once a month. The data and prediction products are sent to the users and relevant communities in China and exchanged with the World Warning Agency (WWA) of IUWDS/ISES as well as other RWCs around the world. WWA is the hub for data and forecasts exchanges in IUWDS/ISES, which is operated by the Space Weather Prediction Center (SWPC), i.e., RWCUSA, in Boulder (ISES, 2017; SWPC, 2017); also note that IUWDS was succeeded by ISES in 1996; see Sect. 1. In the mid-1990s, all four subcenters of RWC-Beijing were using the Internet, and e-mail replaced the telex for daily information exchanges.

With the establishment of RWC-Beijing, the product line of the SAPC at BAO was extended to cover short-term, medium-term, and long-term solar activity predictions. The short-term prediction was issued every weekday and included 1-2-day solar X-ray flare and proton event forecasts (Zhang et al., 1994). The first short-term solar activity prediction of SAPC with standard URSIgram codes was released in January 1991. The medium-term prediction of SAPC was distributed every half-month, and the forecast items included shortwave fadeouts and solar proton events associated with flares and the smoothed monthly mean sunspot number over the next half-month. It was the continuation of the traditional medium-term prediction service of BAO provided since 1969 (see Sect. 1). The long-term prediction of solar activity estimated the smoothed monthly mean sunspot numbers and parameters of the forthcoming solar cycle with a timescale of years, which was made irregularly (Wang, 1993).

Besides the operational prediction services, RWC-Beijing was also active in solar-terrestrial prediction modeling and physics research. Many researchers of RWC-Beijing joined a large Chinese research program entitled Energy Transport Processes in the Solar-Terrestrial System and the follow-up research programs, which have been supported by the National Natural Science Foundation of China (NSFC) since 1993. RWC-Beijing scientists also participated in an international cooperation project called SOLar connections to Transient Interplanetary Processes (SOLTIP), which operated in the 1990s (Wang, 1997).
In the 1990s, RWC-Beijing held several workshops on solar-terrestrial prediction. The first Chinese Workshop of Solar-Terrestrial Prediction was held in 1991 in Suzhou, Jiangsu Province, China. The second Chinese Workshop of Solar-Terrestrial Prediction was held in 1993 in Xi'an, Shaanxi Province, China. The Third Chinese Workshop of Solar-Terrestrial Prediction (together with the Chinese Workshop of Coronal Mass Ejection) was held in 1997 in Dali, Yunnan Province, China. In 1994, members of RWC-Beijing were responsible for organizing the Fourth Chinese SOLTIP Workshop, which was held in Sichuan province, China (Wang, 1997). Scientists of RWC-Beijing also attended several international meetings organized by IUWDS/ISES in the 1990s, such as the meeting in Canada in 1992 and the meeting in Japan in 1996.

Personnel exchanges and collaborations with other RWCs were facilitated at RWC-Beijing. In the 1990s, members of RWC-Beijing visited RWC-Russia (Moscow), RWCAustralia (Sydney), RWC-Japan (Tokyo), and RWC-USA (Boulder). Meanwhile, scientists of RWC-Japan, RWCAustralia, RWC-Russia, and RWC-USA also made successful visits to RWC-Beijing (Wang, 1997). Scientific exchanges were implemented through these mutual visits.

In addition to prediction services, data services, and collaborative research services for professional users (e.g., scientists, satellite operators, satellite orbit forecasters, shortwave communicators, power plant and electrical equipment maintainers, etc.), RWC-Beijing also provided services for external users, such as weather and climate forecasters, harvest forecasters, disaster forecasters, geophysical explorers, and amateurs. RWC-Beijing tried to provide users with a simple, clear, and quantitative service that would be useful for their own purposes (Wang, 1997). After 10 years of development in the 1990s, RWC-Beijing had laid a solid foundation for its role in solar-terrestrial prediction services and collaborations in China.

\section{RWC-China in the new century}

With the coming of the 21 st century, human life is more and more dependent on electronic devices and space satellites (e.g., satellite communications and GPS applications); the industrial automation and control systems in factories are more sensitive to geomagnetic disturbances, and the concept of space weather is generally known by the public. Economic development in China provided more opportunities for space weather research, operation, and instrument construction. Solar-terrestrial prediction services entered a new era.

In 2000, RWC-Beijing was renamed RWC-China according to ISES requirements. In 2001, the National Astronomical Observatories, CAS (NAOC) was established based on the BAO and several other astronomical institutes of CAS. All the solar-terrestrial data and prediction services of BAO were taken up by NAOC. The headquarters of RWC- 
Table 1. Four subcenters of RWC-China.

\begin{tabular}{llll}
\hline Subcenter name & Service area & $\begin{array}{l}\text { Web page for opera- } \\
\text { tional prediction (2018) }\end{array}$ & Host institution \\
\hline $\begin{array}{l}\text { Solar Activity Prediction } \\
\begin{array}{l}\text { Center (SAPC) } \\
\text { (headquarters of RWC-China) }\end{array}\end{array}$ & $\begin{array}{l}\text { Solar activity } \\
\text { prediction }\end{array}$ & http://rwcc.bao.ac.cn/ & $\begin{array}{l}\text { National Astronomical Obser- } \\
\text { vatories, CAS (NAOC) } \\
\text { http://www.nao.cas.cn/ }\end{array}$ \\
\hline $\begin{array}{l}\text { Space Environment Prediction } \\
\text { Center (SEPC) }\end{array}$ & $\begin{array}{l}\text { Space environment } \\
\text { prediction }\end{array}$ & http://www.sepc.ac.cn/ & $\begin{array}{l}\text { National Space Science Center, } \\
\text { CAS (NSSC) } \\
\text { http://www.nssc.cas.cn/ }\end{array}$ \\
\hline $\begin{array}{l}\text { Ionospheric Disturbance } \\
\text { Prediction Center (IDPC) }\end{array}$ & $\begin{array}{l}\text { Ionospheric } \\
\text { disturbance } \\
\text { prediction }\end{array}$ & (via e-mail) & $\begin{array}{l}\text { China Research Institute of Ra- } \\
\text { diowave Propagation (CRIRP) } \\
\text { http://www.crirp.ac.cn/ }\end{array}$ \\
\hline $\begin{array}{l}\text { Geomagnetic Storm Prediction } \\
\text { Center (GSPC) }\end{array}$ & $\begin{array}{l}\text { Geomagnetic storm } \\
\text { prediction }\end{array}$ & (via e-mail) & $\begin{array}{l}\text { Institute of Geology and Geo- } \\
\text { physics, CAS (IGG) } \\
\text { http://www.igg.cas.cn/ }\end{array}$ \\
\hline
\end{tabular}

China is located on the campus of NAOC. The four subcenters of RWC-China now include the Solar Activity Prediction Center (SAPC), the Space Environment Prediction Center (SEPC), the Ionospheric Disturbance Prediction Center (IDPC), and the Geomagnetic Storm Prediction Center (GSPC), which are hosted by the NAOC, the National Space Science Center (NSSC), the China Research Institute of Radiowave Propagation (CRIRP), and the Institute of Geology and Geophysics (IGG), respectively. (See Table 1 for a summary of the four subcenters, and note a minor adjustment to the subcenter names of IDPC and GSPC in contrast to the RWC-Beijing era; the service areas and the current web pages of the subcenters are also given in Table 1.) Meanwhile, more institutions and colleges have become involved in the space weather community in China (Wei et al., 2003; Wang and Wang, 2006). In particular, the China Meteorological Administration (CMA) contributes to the discussion of space weather in the World Meteorological Organization (WMO) and the International Civil Aviation Organization (ICAO) as a representative of China; the Polar Research Institute of China (PRIC) also has a wealth of activities related to space weather research. The efforts of the whole space weather community provide valuable observational and scientific support for the operational activities of RWC-China (Wang and Wang, 2006).

At the beginning of the new century, several new solarterrestrial observation instruments and projects were proposed and constructed. In 2005, a new solar magnetic field telescope, the Solar Magnetism and Activity Telescope (SMAT) was set up at HSOS of NAOC (Zhang et al., 2007) and can obtain full disk photospheric vector magnetic field data of the Sun. In 2010, the $1 \mathrm{~m}$ New Vacuum Solar Telescope (NVST) was set up at the Fuxian Solar Observatory of YNAO and put into use (Liu et al., 2014). The antenna array of the MingantU SpEctral Radioheliograph (MUSER) of NAOC, formerly known as the Chinese Spectral Radioheli-

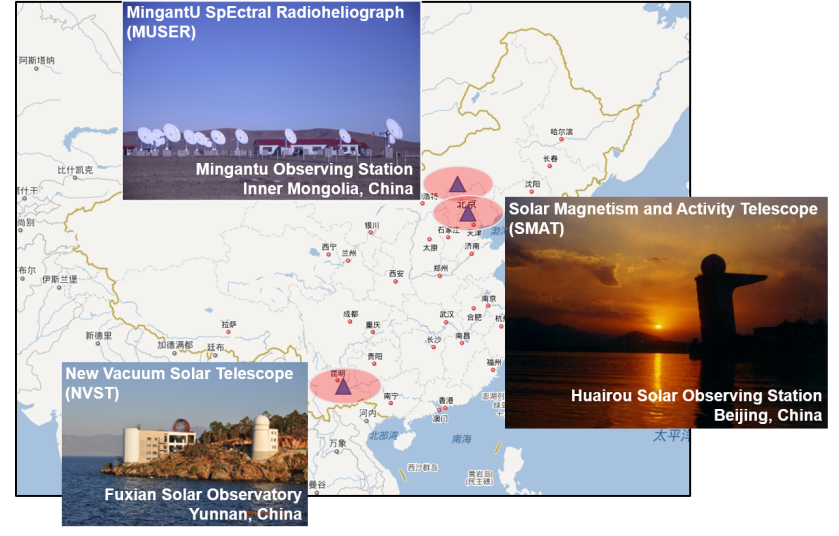

Figure 2. A map of the three solar observation instruments SMAT, NVST, and MUSER constructed in the new century.

\section{lonosonde and digisonde network operated by IDPC}

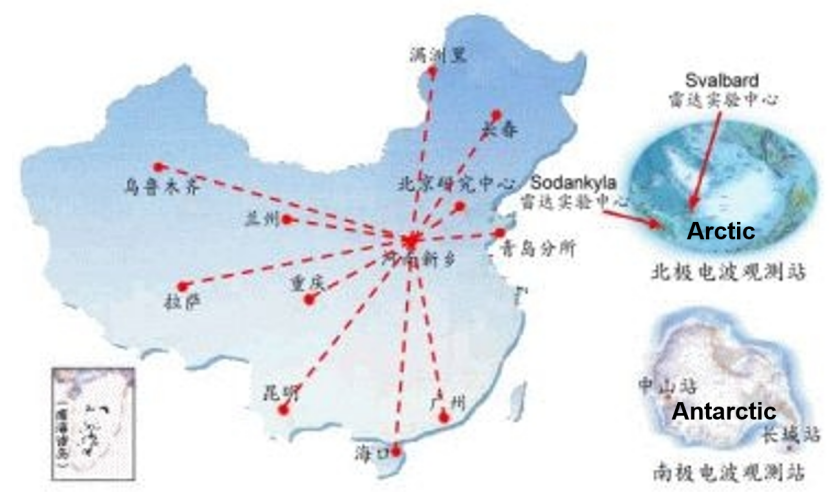

Figure 3. A map of the ionosonde and digisonde network operated by the IDPC. 


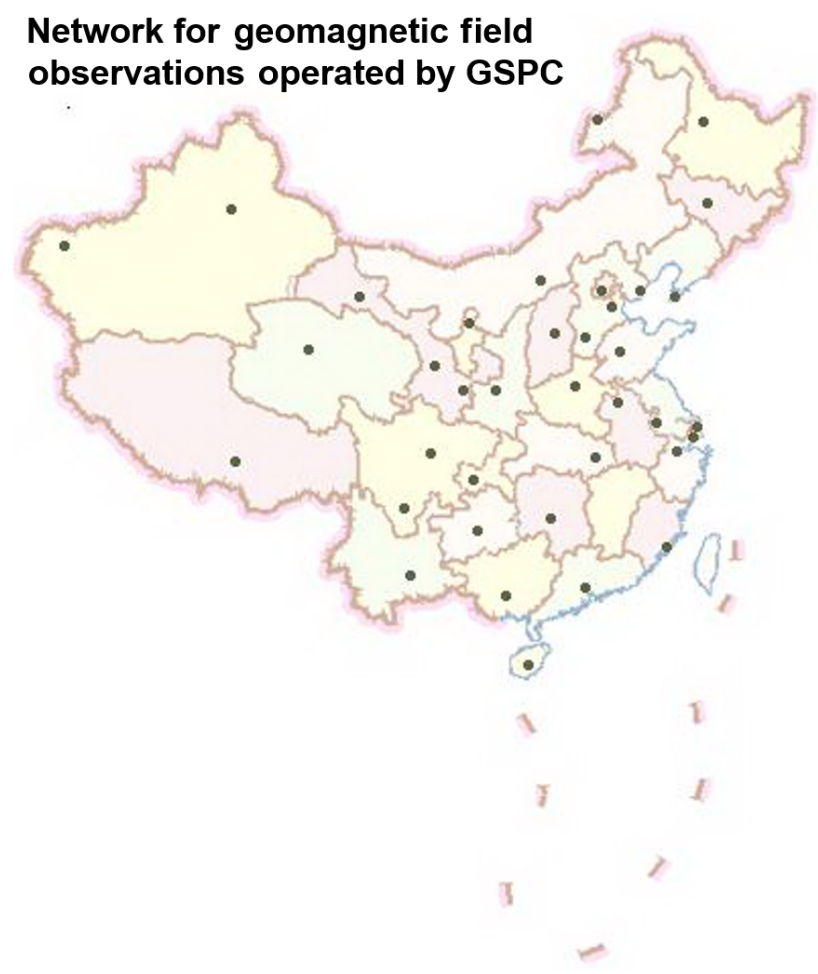

Figure 4. A map of the network for geomagnetic field observations operated by the GSPC.

ograph (CSRH), has been constructed at the Mingantu Observing Station in Inner Mongolia, northern China and put into operational observation (Yan et al., 2009). (See Fig. 2 for a map of the three solar observation instruments.) An ionosonde and digisonde network connecting most of the cities in China and several stations in the Arctic and Antarctic has been constructed and is operated by the IDPC of RWCChina. A large-scale network for geomagnetic field observations has also been constructed and is operated by the GSPC of RWC-China (Wang and Wang, 2006). (See Figs. 3 and 4 for maps of the observation networks operated by the IDPC and GSPC.) A new ground-based space weather monitoring instrument network for monitoring China's geospace environment, the Meridian Space Weather Monitoring Project (or Chinese Meridian Project), has been constructed and started to collect data in 2012. It consists of a chain of 15 ground-based observatories roughly along $120^{\circ} \mathrm{E}$ longitude and $30^{\circ} \mathrm{N}$ latitude. (A map of the 15 observatories can be found in the article by Wang, 2010a.) All four institutes that host the four subcenters of RWC-China (see Table 1) participate in the project (Wang, 2010a).

Besides the usual routine services of solar-terrestrial data and predictions, in the new century, members of RWC-China also provide special space weather prediction services for several Chinese space missions, especially for the Shenzhou series of manned space flights and the Chang'e series of moon exploration spacecrafts (Wang, 2010b). Figure 5 shows

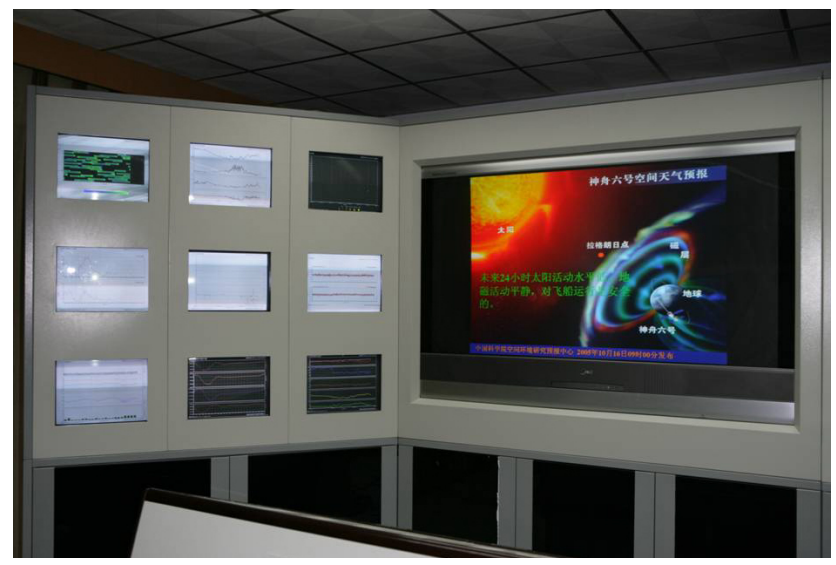

Figure 5. Space weather prediction service at SEPC of RWC-China for the Chinese Shenzhou VI manned space flight mission in October 2005 .

an example of these services, which is for the Chinese Shenzhou VI manned space flight mission in October 2005. These special services (presented by the SEPC and contributed by all members of RWC-China) are effective and successful and have been praised by users. The space environment data detected by the spacecrafts also enriched the data collections of RWC-China.

In 2000, a new series of basic research programs on space weather was initiated in China and is supported by the Ministry of Science and Technology of China. The first program of this series was named Intense Solar Activities and Space Weather Hazards. Scientists of RWC-China were involved in the programs and made great efforts to improve the forecasting models. Many papers on space weather modeling were published in domestic and international journals. The contents of the model studies cover a wide range of topics including solar activity, interplanetary space environment, geomagnetic activity, ionosphere, and atmosphere environment. The main purpose of the research programs is to integrate different models to form a chain of forecasting from the Sun to the Earth (Wang and Wang, 2006; Wang, 2010b).

At SAPC, artificial intelligence techniques and physical analyses of the photospheric magnetic field and coronal magnetic field have been introduced in solar activity prediction model studies in recent years (Wang et al., 2008; He et al., 2008). The currently available prediction models for the operational forecasting of solar activities include the solar flare short-term prediction model, the solar proton event shortterm prediction model, the solar $10.7 \mathrm{~cm}$ radio flux prediction model, the solar active level quantitative assessment model, the nonlinear force-free field (NLFFF) extrapolation model for the coronal magnetic field, and the solar cycle long-term prediction model (He et al., 2012). In addition to solar X-ray flare and proton event forecasting, solar $10.7 \mathrm{~cm}$ radio flux daily value forecasting for the following 3 days was added to the routine services of short-term prediction at SAPC. In 


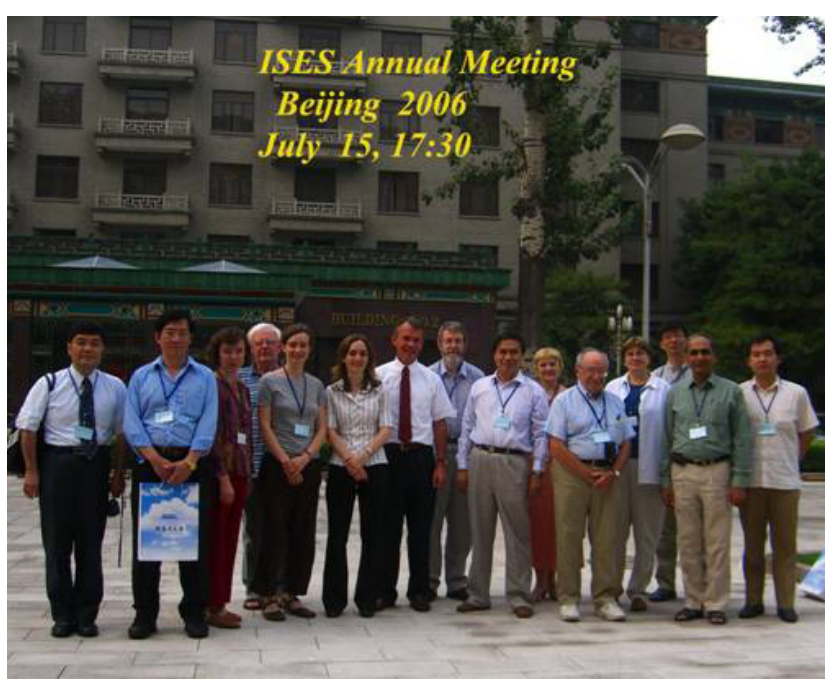

Figure 6. ISES Annual Meeting 2006 at Beijing Friendship Hotel on 15 July 2006.

2010, the solar X-ray flare active level forecasting for the next 7 days ( 1 week) was added to the medium-term prediction services. A verification of the short-term solar Xray flare predictions issued by RWC-China in the maximum phase (2000-2001) of solar cycle 23 gives an accuracy rate of $66 \%$, which is similar to the predictions by the WWA of ISES (Zhu and Wang, 2003).

International and domestic scientific meetings on space weather were frequently held in China during the past decades. Personnel exchanges and cooperation between RWC-China and other RWCs are more frequent than ever. In July 2006, the 36th COSPAR Scientific Assembly was held in Beijing, China. Taking this opportunity, RWC-China hosted the ISES Annual Meeting 2006 at Beijing Friendship Hotel on 15 July 2006. The group photo of this meeting is shown in Fig. 6. In 2009, also in Beijing, RWC-China held a domestic workshop for space weather prediction modeling and application.

In recent years, Internet applications are more and more important for scientific activities. Many discussions and cooperation now can be done through Internet tools. Computer platforms and Internet services (e.g., web pages) play an important role in the operational activities of space weather monitoring, data exchanges, and forecast distributions (see Table 1 for Internet services employed by the subcenters of RWC-China). In 2000, RWC-China began to develop a computer platform system and web pages (http://rwcc.bao.ac.cn/) for operational space weather predictions. Until now, there have been three generations of operational platforms that were developed or are being developed at RWC-China (He et al., 2012). The first-generation platform was a single machine system with a simple database and web pages, which was put into use during 2001-2006. The second-generation platform was a multi-machine system, which was put into use after 2006. It has complex data tables in the database system, with the prediction models being integrated into the platform. The third-generation platform is being developed at RWCChina and has a dedicated database server and mass storage device with interactive web pages and a 3-D computer simulation interface. The third-generation system is a completely new operational platform for space weather monitoring and prediction and will be the basis for a new starting point of RWC-China for future advances in the 21 st century.

\section{Summary}

After 25 years of development, RWC-China has established a complete service system of space weather predictions, developed certain prediction models for operational forecasting, and constructed computer systems and Internet services for data exchange and forecast distribution. Domestic and international cooperation is carried out regularly and intensively. Besides the NAOC having been the representative institution of RWC-China, the host institutions of the other three subcenters (see Table 1) were recently assigned as the Associate Warning Centers (AWCs) of ISES (ISES, 2017), which means that all the subcenters of RWC-China are playing a more active role in the ISES community.

In the future, while still emphasizing services for traditional professional and external users, RWC-China will pay more attention to services for ordinary people, since the effects of space weather disturbances have gradually reached into everyday life. More physical mechanism analyses will be introduced in space weather prediction model studies in addition to the traditional statistical approaches and the experiences of the forecasters. Quantitative prediction models will be developed to provide users with more precise forecasts. Data collection and automatic analyses will greatly rely on computer systems, and the results of quantitative forecasts will be presented to the user with visualized scenes and 3-D simulations. The products of ST predictions will be more suitable for the real needs of users. In addition to the current approaches to international scientific exchanges, RWC-China will devote itself to international cooperation projects and provide contributions to the further development of ISES.

Data availability. No data sets were used in this article.

Competing interests. The authors declare that they have no conflict of interest.

Special issue statement. This article is part of the special issue "History of regional warning centers". It is not associated with a conference. 
Acknowledgements. The authors are supported by the National Science and Technology Basic Work Program of China (grant 2014FY120300), the National Natural Science Foundation of China (NSFC) through grants 11603040, 11473040, 11403044, 11272031, 11303051, 11221063, 40890160, 40890161, and 10803011, the National Basic Research Program of China (973 Program) through grant 2011CB811406, and the Strategic Priority Research Program on Space Science, Chinese Academy of Sciences (grant XDA04060801). We also acknowledge the support of the China Meteorological Administration (grant no. GYHY201106011).

Edited by: Philip Wilkinson

Reviewed by: Ishii Mamoru and one anonymous referee

\section{References}

Ai, G. X.: Solar magnetic field telescope, Publications of the Beijing Astronomical Observatory, 9, 27-36, 1987.

Carrington, R. C.: Description of a Singular Appearance seen in the Sun on September 1, 1859, Mon. Not. R. Astron. Soc., 20, 13-15, https://doi.org/10.1093/mnras/20.1.13, 1859.

Green, J. L. and Boardsen, S.: Duration and extent of the great auroral storm of 1859, Adv. Space Res., 38, 130-135, https://doi.org/10.1016/j.asr.2005.08.054, 2006.

He, H., Wang, H. N., Du, Z. L., Li, R., Cui, Y. M., Zhang, L. Y., and He, Y. L.: Solar activity prediction studies and services in NAOC, Adv. Space Res., 42, 1450-1456, https://doi.org/10.1016/j.asr.2007.02.068, 2008.

He, H., Wang, H. N., Du, Z. L., Zhang, L. Y., Huang, X., Yan, Y., Fan, Y. L., Zhu, X. S., Guo, X. B., and Dai, X. H.: Recent progress of solar weather forecasting at NAOC, in: Advances in Geosciences, Proceedings of the 8th Annual Meeting of the Asia Oceania Geosciences Society, Taipei, 8-12 August 2011, 30, 117-126, https://doi.org/10.1142/9789814405744_0008, 2012.

ISES: International Space Environmental Service, available at: http: //www.ises-spaceweather.org/ or http://www.spaceweather.org/, last access: 20 November 2017.

Lanzerotti, L. J.: Space Weather: Historical and Contemporary Perspectives, Space Sci. Rev., 212, 1253-1270, https://doi.org/10.1007/s11214-017-0408-y, 2017.

Liu, Z., Xu, J., Gu, B.-Z., Wang, S., You, J.-Q., Shen, L.-X. Lu, R.W., Jin, Z.-Y., Chen, L.-F., Lou, K., Li, Z., Liu, G.-Q., Xu, Z., Rao, C.-H., Hu, Q.-Q., Li, R.-F., Fu, H.-W., Wang, F., Bao, M.X., Wu, M.-C., and Zhang, B.-R.: New vacuum solar telescope and observations with high resolution, Res. Astron. Astrophys., 14, 705-718. https://doi.org/10.1088/1674-4527/14/6/009, 2014.

NAOC: History of Beijing Astronomical Observatory, Chinese Academy of Sciences (1958-2001), China Science and Technology Press, Beijing, China, 2009.

SWPC: Space Weather Prediction Center, available at: http://www. swpc.noaa.gov/, latest access 20 November 2017.
URSI: International Union of Radio Science, available at: http:// www.ursi.org/, last access: 20 November 2017.

URSIgram: URSIgram Codes, available at: http://www. spaceweather.org/ISES/code/code.html, last access: 20 November 2017.

Wang, C.: New Chains of Space Weather Monitoring Stations in China, Space Weather, 8, S08001, https://doi.org/10.1029/2010SW000603, 2010a.

Wang, H. N.: RWC-China $\mathrm{R}_{2} \mathrm{O}$ activities: progresses and challenges: 38th COSPAR Scientific Assembly, Bremen, Germany, 18-25 July 2010, PSW1-0015-10, 2010b.

Wang, H. N., Cui, Y. M., Li, R., Zhang, L. Y., and Han, H.: Solar flare forecasting model supported with artificial neural network techniques, Adv. Space Res., 42, 1464-1468, https://doi.org/10.1016/j.asr.2007.06.070, 2008.

Wang, H. N. and Wang, J. X.: Space weather study in China: ISES Annual Meeting 2006, Beijing, China, 15 July 2006.

Wang, J. L.: A summary of solar physics activities in China, in: Solar and Stellar Coronal Structure and Dynamics, Proceedings of the Ninth Sacramento Peak Summer Symposium, Sunspot, NM, 17-21 August 1987, 292-295, 1988.

Wang, J. L.: Solar activity predictions at RWC-Beijing, in: SolarTerrestrial Predictions - IV, Proceedings of a Workshop at Ottawa, Canada, 18-22 May 1992, vol. I, 278-285, 1993.

Wang, J. L.: RWC-Beijing activities report, 1993-1995, in: SolarTerrestrial Predictions - V, Proceedings of a Workshop at Hitachi, Japan, 23-27 January 1996, 34-36, 1997.

Wei, F., Feng, X., Guo, J.-S., Fan, Q., and Wu, J.: Space Weather Research in China, Space Sci. Rev., 107, 327-334. https://doi.org/10.1023/A:1025525201204, 2003.

Yan, Y., Zhang, J., Wang, W., Liu, F., Chen, Z., and Ji, G.: The Chinese Spectral Radioheliograph - CSRH, Earth Moon Planets, 104, 97-100, https://doi.org/10.1007/s11038-008-9254-y, 2009.

Zhang, G. Q., Wang, J. L., and Li, D. Q.: A new scheme used for the short-term prediction of X-ray flares, Publications of the Beijing Astronomical Observatory, 24, 24-31, 1994.

Zhang, H. Q., Wang, D. G, Deng, Y. Y., Hu, K. L., Su, J. T., Lin, J. B., Lin, G. H., Yang, S. M., Mao, W. J., Wang, Y. N., Hu, Q. Q., Xue, J. S., Lu, H. T., Ni, H. K., Chen, H. L., Zhou, X. J., Zhu, Q. S., Yuan, L. J., and Zhu, Y.: Solar Magnetism and the Activity Telescope at HSOS, Chinese J. Astron. Ast., 7, 281-288, https://doi.org/10.1088/1009-9271/7/2/12, 2007.

Zhang, M., Deng, Y. Y., and Zhang, H. Q.: Vector magnetic field measurement at Huairou and helicity of active regions, Adv. Space Res., 39, 1741-1748, https://doi.org/10.1016/j.asr.2007.03.044, 2007.

Zhu, C. L. and Wang, J. L.: Verification of short-term predictions of solar soft X-ray bursts for the maximum phase (20002001) of solar cycle 23, Chinese J. Astron. Ast., 3, 563-568, https://doi.org/10.1088/1009-9271/3/6/563, 2003. 